\title{
THE SYMBOLIC REPRESENTATION OF BILLIARDS WITHOUT BOUNDARY CONDITION
}

\author{
TAKEHIKO MORITA
}

\begin{abstract}
We consider a dynamical system with elastic reflections in the whole plane and show that such a dynamical system can be represented as a symbolic flow over a mixing subshift of finite type. This fact enables us to prove an analogue of the prime number theorem for the closed orbits of such a dynamical system.
\end{abstract}

\section{INTRODUCTION}

Let $O_{1}, O_{2}, \ldots, O_{L}(L \geq 3)$ be a finite number of bounded domains in $\mathbb{R}^{2}$ with smooth boundaries $\partial O_{1}, \partial O_{2}, \ldots, \partial O_{L}$. We assume that the closures $\overline{O_{j}}=O_{j} \cup \partial O_{j}$ of $O_{j}$ are strictly convex and mutually disjoint. Consider the motion of a particle in the exterior domain $O=\mathbb{R}^{2} \backslash \bigcup_{j=1}^{L} \overline{O_{j}}$, which obeys the law of reflection: "the particle moves along the straight line with unit speed in $O$ and reflects at the boundary $\partial O=\bigcup_{j=1}^{L} \partial O_{j}$ so that the angle of the reflection coincides with the angle of the incidence." We can describe this motion of a particle by a dynamical system (a flow) $S_{t}$ on the unit tangent bundle over $O$. We call the flow $S_{t}$ a billiard without boundary condition in the light of the Sinai's billiard in [6], which is defined on the unit tangent bundle over 2-torus $T^{2}$, i.e., the billiard with periodic boundary condition.

The purpose of this paper is to prove the following theorems:

Theorem 1. Under the hypotheses (H.1) and (H.2) (see §2), the flow $S_{t}$ restricted to the nonwandering set can be represented as a symbolic flow $\sigma_{t}$ over an appropriate subshift of finite type so that the corresponding closed orbits have the same period (see Proposition 3.1).

Theorem 2. Under the hypotheses (H.1) and (H.2), there is a positive constant $h$ such that an analogue of the prime number theorem

$$
\#\left\{\gamma ; \exp \left[h T_{\gamma}\right] \leq x\right\} \cdot \frac{\log x}{x} \rightarrow 1 \quad(x \rightarrow \infty)
$$

holds, where $\gamma$ and $T_{\gamma}$ denote the prime closed orbit of $S_{t}$ and its period respectively.

Received by the editors May 12, 1989.

1980 Mathematics Subject Classification (1985 Revision). Primary 58F20, 58F15; Secondary 58F40, 70D99. 
It is not hard to see that Theorem 2 is obtained by combining Theorem 1 and the result in Parry and Pollicott [5] (see $\S 3$ ).

It will be meaningful to note that the closed orbits of the dynamical system $S_{t}$ make the essential contribution to the singular support of the distributional function $\sum_{\lambda_{i} \in \operatorname{Spec} \Delta} \cos \lambda_{i}^{1 / 2} t$ (see [1]) and they are closely related to the poles of the scattering matrix as mentioned in Ikawa [2] and [3] etc., where $\operatorname{Spec} \Delta$ denotes the set of eigenvalues of the selfadjoint realization of the Laplace operator with the appropriate boundary condition.

The author would like to express his sincere gratitude to Professor M. Ikawa for introducing him to such an interesting topic. He would also like to express his thanks to Professor M. Wada for his helpful suggestions on the symmetric orbits of the flow $S_{t}$.

\section{Preliminaries}

In this section we prepare the basic notions for the later convenience.

Let $O_{1}, O_{2}, \ldots, O_{L}(L \geq 3)$ be a finite number of bounded domains in $\mathbb{R}^{2}$ as in the beginning of Introduction. We denote by $S \mathbb{R}^{2}=\mathbb{R}^{2} \times S^{1}=\{(q, v) \in$ $\left.\mathbb{R}^{2} \times \mathbb{R}^{2} ;|v|=1\right\}$ the unit tangent bundle over $\mathbb{R}^{2}$ and $\pi: S \mathbb{R}^{2} \rightarrow \mathbb{R}^{2}$ the natural projection, where $|\cdot|$ denotes the usual Euclidean norm. Choose a point $q_{j} \in \partial O_{j}$ for each $j$ and define the following quantities for $x=(q, v) \in$ $\partial O=\bigcup_{j=1}^{L} \partial O_{j}$ :

$$
\left\{\begin{aligned}
& \xi_{0}(x)=j, \quad \text { if } q \in \partial O_{j} \\
& r(x)= \text { the arclength between } q_{\xi_{0}(x)} \text { and } q, \\
& \text { measured clockwise along the curve } \partial O_{\xi_{0}(x)} \\
& \phi(x)= \text { the angle between the vector } v \text { and the } \\
& \text { unit innernormal } n(q) \text { of } \partial O_{\xi_{0}(x)} \text { at } q \\
& \text { measured unitclockwise. }
\end{aligned}\right.
$$

Therefore $\pi^{-1}(\partial O)$ is parametrized as

$$
\begin{aligned}
& \pi^{-1}(\partial O)=\{(j, r, \phi) ; 1 \leq j \leq L, \\
& \left.0 \leq r<\text { the perimeter of } \partial O_{j} \text {, and } 0 \leq \phi<2 \pi\right\} .
\end{aligned}
$$

Put

$$
\left\{\begin{array}{l}
M_{-}=\left\{x \in \pi^{-1}(\partial O), \quad \frac{\pi}{2} \leq \phi(x) \leq \frac{3}{2} \pi\right\}, \text { and } \\
M_{+}=\left\{x \in \pi^{-1}(\partial O), \quad \frac{\pi}{2}<\phi(x)+\pi<\frac{3}{2} \pi \bmod 2 \pi\right\} .
\end{array}\right.
$$

We introduce the following equivalence relation ' $\sim$ ' to $\pi^{-1}(\partial O)$ :

$$
x \sim y \text { if and only if } \operatorname{Inv}(x)=y \text { or } x=y,
$$

where Inv : $\pi^{-1}(\partial O) \rightarrow \pi^{-1}(\partial O)$ is defined by

$$
\operatorname{Inv}(j, r, \phi)=(j, r, \pi-\phi) \bmod 2 \pi .
$$


It is natural to identify $\left(\pi^{-1}(\partial O)\right) / \sim$ with $M_{-}$and we often use this identification without specification. Put

$$
M=\pi^{-1}(O) \cup\left(\pi^{-1}(\partial O) / \sim\right)=\pi^{-1}(O) \cup M_{-} .
$$

Now we recall the notion of the billiard without boundary condition. Consider the motion of a particle which moves along the straight line with unit speed in $O=\mathbb{R}^{2} \backslash \bigcup_{j=1}^{L} \bar{O}_{j}$ and reflects at the boundary $\partial O=\bigcup_{j=1}^{L} \partial O_{j}$ according to the law of reflection: the angle of reflection coincides with that of incidence. Then the motion determines a dynamical system (a flow) on $M$ in a canonical way (see Remark 1.2 below). We call it a billiard without boundary condition.

Remark 1.1. It is easy to see that $M_{-}$and $M_{+}$denote the set of the incidental vectors and the set of the reflection vectors respectively.

We define the first collision time $\tau_{+}$and the last collision time $\tau_{-}$by

$$
\left\{\begin{array}{l}
\tau_{+}(x)=\inf \left\{t>0, \pi\left(S_{t} x\right) \in \partial O\right\} \\
\tau_{-}(x)=\sup \left\{t<0, \pi\left(S_{t} x\right) \in \partial O\right\}
\end{array}\right.
$$

Here we regard $\tau_{+}(x)$ (resp. $\tau_{-}(x)$ ) as $+\infty$ (resp. $-\infty$ ) if the set in the definition is empty.

Remark 1.2. Let $x=(q, v), M=\pi^{-1}(O) \cup M_{-}$. We note that the flow $S_{t}$ is defined so that

$$
S_{t} x= \begin{cases}(q+t v, v), & \left(x \in \pi^{-1}(O)\right) \\ (q+t \tilde{v}, \tilde{v}), & \left(x \in M_{-}\right)\end{cases}
$$

if $0<t<\tau_{+}(x)$, where $\tilde{v}$ is determined by the formula $\operatorname{Invx}=(q, \tilde{v})$.

Put

$$
\begin{aligned}
\Omega=\left\{x \in M, \pi\left(S_{t} x\right) \in \partial O\right. \text { for both infinitely many } \\
\qquad t>0 \text { and infinitely many } t<0\} .
\end{aligned}
$$

Clearly, $\Omega$ coincides with the nonwandering set of the flow $S_{t}$. Put

$$
\Omega_{0}=\pi^{-1}(O) \cap \Omega \text { and } \Omega_{-}=M_{-} \cap \Omega .
$$

We define the local maps $T$ and $T^{-1}$ by

$$
\left\{\begin{array}{l}
T(x)=S_{\tau_{+}(x)}(x) \quad \text { if } \tau_{+}(x)<+\infty, \\
T^{-1}(x)=S_{\tau_{-}(x)}(x) \quad \text { if } \tau_{-}(x)>-\infty,
\end{array}\right.
$$

respectively.

It is not hard to see that the above notation $T^{-1}$ is compatible with the definition of the inverse map of $T$ and $T$ is locally diffeomorphic.

Remark 1.3. Consider the flow $S_{t}$ restricted to $\Omega$. The set $\Omega_{-}$and the first collision time $\tau_{+}$play the role of the Poincare section and the Poincare map respectively. 
For $x \in M_{-}\left(=\pi^{-1}(\partial O) / \sim\right)$, we put

$$
\xi_{j}(x)=\xi_{0}\left(T^{j} x\right) \text { if } T^{j} \text { is defined. }
$$

We call the sequence $\xi=\left(\xi_{j}\right)_{j=-\infty}^{\infty}$ the itinarary of $x \in \Omega_{-}$if $\xi_{j}=\xi_{j}(x)$ and write $\xi$ as $\xi(x)$.

We conclude this section by stating the following lemma. The proof is due to elementary calculation of the Jacobi matrix of $T$, and it can be found in [4]. Therefore we omit the proof.

Lemma 1.1. Let $C$ be a curve of class $C^{1}$ in $M_{-}$which is represented as $\{(j, r, \phi) ; \phi=\psi(r), a \leq r \leq b\}$, where $\psi$ is a $C^{1}$-function in $r$. Assume that $T$ (resp. $T^{-1}$ ) is defined and continuous on $C$. If the image $C_{1}=T C$ (resp. $\left.C_{-1}=T^{-1} C\right)$ is represented as $\left\{\left(j_{1}, r_{1}, \phi_{1}\right) ; \phi_{1}=\psi_{1}\left(r_{1}\right), a_{1} \leq r_{1} \leq b_{1}\right\}$ (resp. $\left.\left\{\left(j_{-1}, r_{-1}, \phi_{-1}\right) ; \phi_{-}=\psi_{-}\left(r_{-1}\right), a_{-1} \leq r_{-1} \leq b_{-1}\right\}\right)$, where $\psi_{1}$ (resp. $\psi_{-1}$ ) is $C^{1}$-function in $r_{1}$ (resp. $\left.r_{-1}\right)$, then we have:

$$
\begin{aligned}
& \frac{d \psi_{1}}{d r_{1}}=k\left(r_{1}\right)-\frac{\cos \psi_{1}}{\cos \psi}\left(\frac{\tau_{+}(j, r, \phi)}{\cos \psi}-\left(\frac{d \psi}{d r}+k(r)\right)^{-1}\right)^{-1} \\
& \left(\text { resp. } \frac{d \psi_{-1}}{d r_{-1}}=-k\left(r_{-1}\right)-\frac{\cos \psi_{-1}}{\cos \psi}\left(\frac{\tau_{-}(j, r, \phi)}{\cos \psi}-\left(\frac{d \psi}{d r}+k(r)\right)^{-1}\right)^{-1}\right), \\
& \text { (1.13) } \frac{d r_{1}}{d r}=-\frac{\cos \psi}{\cos \psi_{1}}\left(1-\frac{\tau_{+}(j, r, \phi)}{\cos \psi}\left(\frac{d \psi}{d r}+k(r)\right)\right) \\
& \left(\text { resp. } \frac{d r_{-1}}{d r}=-\frac{\cos \psi}{\cos \psi_{-1}}\left(1-\frac{\tau_{-}(j, r, \phi)}{\cos \psi}\left(\frac{d \psi}{d r}-k(r)\right)\right)\right)
\end{aligned}
$$

where $k(r)$ denotes the curvature of $\partial O_{j}$ at $(j, r, \phi)$, etc.

\section{WELL-POSEDNESS OF ITINARARY PROBLEM}

From now on we assume:

(H.1) (convexity). For each $j=1,2, \ldots, L$ boundary $\partial O_{j}$ of $O_{j}$ is a simple closed curve with nonvanishing curvature.

(H.2) (no eclipse). For any triple $\left(j_{1}, j_{2}, j_{3}\right)$ of distinct indices,

$$
\operatorname{conv}\left[O_{j_{1}} \cup O_{j_{2}}\right] \cap O_{j_{3}}=\varnothing,
$$

where $\operatorname{conv}[B]$ denotes the convex hull of the set $B$.

We introduce the following shift dynamical system. Let $A$ be an $L \times L$ matrix with entries $A(i, j)=\left(1-\delta_{i j}\right), 1 \leq i, j \leq L$, where $\delta_{i j}$ denotes the 
Kronecker's delta. Put

$$
\Sigma=\Sigma_{A}=\left\{\xi=\left(\xi_{j}\right)_{j=-\infty}^{\infty} \in \prod_{j=-\infty}^{\infty}\{1,2, \ldots, L\} ;\right.
$$

$$
\left.A\left(\xi_{j}, \xi_{j+1}\right)=1 \text { for any } j\right\}
$$

We define $d_{\rho}: \Sigma \times \Sigma \rightarrow R$ by

$$
d_{\rho}(\xi, \eta)=\rho^{n} \quad \text { if } \xi_{j}=\eta_{j} \text { for }|j|<n \text { and } \xi_{n} \neq \eta_{n} \text { or } \xi_{-n} \neq \eta_{-n} .
$$

It is easy to see that $d_{\rho}$ becomes a metric on $\Sigma$ which defines the same topology as the induced topology of $\Sigma$ as a subset of the product space

$$
\prod_{j=-\infty}^{\infty}\{1,2, \ldots, L\} \text {. }
$$

The shift transformation $\sigma: \Sigma \rightarrow \Sigma,(\sigma \xi)_{j}=\xi_{j+1}, j \in Z$ is well defined and the shift dynamical system $(\Sigma, \sigma)$ is a typical example of a mixing subshift of finite type (see [5]). Consider the billiard without boundary condition $S_{t}$. We say that a point $x \in \Omega_{\text {_ }}$ solves the itinarary problem

$$
\xi(y)=\xi \in \Sigma
$$

or $x$ is a solution of the itinarary problem (2.3) if the itinarary $\xi(x)$ of $x$ coincides with the sequence $\xi$.

Under the hypotheses (H.1) and (H.2) we prove that the itinarary problem (2.3) is well-posed in the following sense.

Theorem 0 (the Lipschitz well-posedness of the itinarary problem). If the hypotheses (H.1) and (H.2) are satisfied, there exists a unique $x \in \Omega_{-}$which solves the itinarary problem (2.3) for any $\xi \in \Sigma$. In addition, if we denote by $x(\xi)$ the solution of the itinarary problem (2.3), there exist constants $C>0$ and $0<\rho<1$ such that

$$
\left|\tau_{+}(x(\xi))-\tau_{+}(x(\eta))\right|<C d_{\rho}(\xi, \eta) \text { for any } \xi, \eta \in \Sigma,
$$

where $d_{\rho}$ denotes the metric on $\Sigma$ defined by (2.2).

We prepare an a priori estimate for the proof of Theorem 0.

Lemma 2.1 (a priori estimate). Let $x$ and $y$ be elements in $M_{-}$. Assume $T^{j}$ is well defined and $\xi_{j}(x)=\xi_{j}(y)$ for each $j$ with $-n \leq j \leq n \quad(n \geq 1)$. Then the arclength $r(x, y)$ between $\pi(x)$ and $\pi(y)$ satisfies

$$
r(x, y) \leq c_{0} l(1+\eta)^{-n}
$$

where $c_{0}$ is a positive constant independent of $x$ and $y$,

$$
\begin{aligned}
l= & \max \left\{\text { the perimeter of } \partial O_{j}, j=1,2, \ldots, L\right\} \text { and } \\
\eta= & \min \left\{\text { the distance between } \overline{O_{i}} \text { and } \overline{O_{j}}, 1 \leq i<j \leq L\right\} \\
& \times \min \left\{k(q), q \leq \partial O_{j}, j=1,2, \ldots, L\right\} .
\end{aligned}
$$


Proof. Let $C$ be a $C^{1}$-curve in $M_{-}$as in Lemma 1.1. We call it an increasing (resp. decreasing) curve if $\frac{d \psi}{d r} \geq 0$ (resp. $\frac{d \psi}{d r} \leq 0$ ). Assume that $x, y \in M_{-}$satisfy the assumption of Lemma 2.1. Then we may write $T^{j} x=\left(r_{j}(x), \phi_{j}(x)\right)$, $j=-n,-(n-1), \ldots, n-1, n$ without confusion. First we connect $x$ and $y$ by a line segment $C_{0}$ in $M_{-}$. We may assume that $r_{0}(x)<r_{0}(y)$. If $\phi_{0}(x) \leq \phi_{0}(y), C_{0}$ becomes an increasing curve in $M_{-}$. Therefore it is not hard to show that $T^{j}$ is continuous on $C_{0}$ and the image $C_{j}=T^{j} C_{0}$ turns out to be an increasing curve for each $j=1,2, \ldots, n$ in the same way as in the proof of Lemma 4.1 in [4]. Thus $C_{j}$ can be expressed as $\left\{\left(r_{j}, \phi_{j}\right)\right.$, $\left.\phi_{j}=\psi_{j}\left(r_{j}\right), a_{j} \leq r_{j} \leq b_{j}\right\}$ with $d \psi_{j} / d r_{j} \geq 0$ for each $j$. In virtue of the formula (1.13), we obtain

$$
\frac{d r_{n}}{d r_{0}}=\frac{d r_{n}}{d r_{n-1}} \frac{d r_{n-1}}{d r_{n-2}} \cdots \frac{d r_{1}}{d r_{0}}=(-1)^{n} \frac{\cos \psi_{n}}{\cos \psi_{0}} \prod_{j=1}^{n} b_{j},
$$

where $b_{j}=\left(1-\tau_{+}\left(T^{j}\left(r_{0}, \phi_{0}\right)\right)\left(d \psi_{j} / d r_{j}+k\left(r_{j}\right)\right)\right.$. Since $d \psi_{j} / d r_{j} \geq 0$ for all $j=0,1, \ldots, n-1$, we have

$$
\left|b_{j}\right| \geq 1+\eta
$$

from the formula (1.12). Thus we have

$$
\left|\frac{d r_{n}}{d r_{0}}\right| \geq\left|\cos \phi_{0}\right|(1+\eta)^{n}
$$

Therefore we obtain

$$
r(x, y) \leq\left|\cos \phi_{0}\right|^{-1} l(1+\eta)^{-n} .
$$

On the other hand it is easy to show that $\left|\cos \phi_{0}\right|$ is bounded below by a positive constant which is independent of $x$ and $y$ in virtue of the hypotheses (H.1) and (H.2). Hence we have proved the inequality (2.5) when $\phi_{0}(x) \leq$ $\phi_{0}(y)$. If $\phi_{0}(x)>\phi_{0}(y)$, we can prove the estimate (2.5) in the same manner, by using $T^{j},-n \leq j \leq-1$, instead of $T^{j}, 1 \leq j \leq n$.

Now we can prove Theorem 0 . The Lipschitz continuity (2.4) of the first collision time is an immediate consequence of Lemma 2.1 if we take $(1+\eta)^{-1}$ as $\rho$. The uniqueness of the itinarary problem follows from the estimate (2.4). Therefore it suffices to show the existence. First we assume that $\xi$ is periodic, i.e., $\xi_{n+m}=\xi_{n}$ for some $m>0$, for all $n \in Z$. Consider the following minimal value problem

$$
\begin{aligned}
& l\left(q^{0}, q^{1}, \ldots, q^{m-1}\right)=\sum_{j=0}^{m-1}\left|q^{j}-q^{j-1}\right| \\
& q^{j} \in \partial O_{\xi_{j}}, j=0,1, \ldots, m-1,
\end{aligned}
$$

where $q^{-1}=q^{m-1}$. 
Hypotheses (H.1) and (H.2) imply that there exists $\left(p^{0}, p^{1}, \ldots, p^{m-1}\right)$ which minimizes $l\left(q^{0}, q^{1}, \ldots, q^{m-1}\right)$ in virtue of the Borzano Weierstrass theorem. The points $p^{0}, p^{1}, \ldots, p^{m-1}$ have to satisfy the equations

$$
\frac{\partial l}{\partial q_{k}^{j}}=\lambda_{j} \frac{\partial f_{j}}{\partial q_{k}^{j}}\left(p^{j}\right), \quad j=0,1, \ldots, m-1 \text { and } k=1,2, \ldots,
$$

where the curves $\partial O_{\xi_{j}}$ are assumed to be represented as $f_{j}\left(q^{j}\right)=0$ in the neighborhood of $p^{j}$, and $\lambda_{j}$ denote the Lagrange multipliers.

The equations (2.11) are nothing but the law of reflections. Therefore the existence of the solution of the itinarary problem has been proved when $\xi$ is periodic.

Let $\xi$ be an element in $\Sigma$ which is not periodic. Choose $\xi^{m} \in \Sigma$ which is periodic and $d_{\rho}\left(\xi^{m}, \xi\right) \rightarrow 0,(m \rightarrow \infty)$. Let $x^{m}$ be the unique solution of the itinarary problem $\xi(x)=\xi^{m}$. The estimate (2.5) in Lemma 2.1 implies that

$$
r\left(T^{j} x^{m}, T^{j} x^{m+1}\right) \leq C \rho^{\left(\text {the period of } \xi^{m}\right)-|j|}
$$

for $|j| \leq$ the period of $\xi^{m}$. Therefore $x^{m}$ converges to some $x \in M_{-}$and $x$ satisfies $\xi(x)=\xi$. Now the proof of Theorem 0 is complete.

Remark 2.1. It is not necessary to use the inequality (2.5) to show the existence of the solution of the itinarary problem. One can show it by use of the diagonal argument.

\section{Proofs of Results}

The purpose of this section is to complete the proofs of Theorem 1 and Theorem 2 in Introduction. Note that we always assume the hypotheses (H.1) and (H.2).

Define a function $f$ on $\Sigma$ by

$$
f(\xi)=\tau_{+}(x(\xi)), \quad \text { for } \xi \in \Sigma,
$$

where $x(\xi)$ denotes the unique solution of the itinarary problem (2.3) as before. We denote by $\left(\Sigma^{f}, \sigma_{t}\right)$ (simply $\sigma_{t}$ ) the symbolic flow over $\sum$ with ceiling function $f$. Precisely, $\Sigma^{f}$ is the set $\{(\xi, s) ; \xi \in \Sigma, 0 \leq s<f(\xi)\}$ with the identification $(\xi, f(\xi))=(\sigma \xi, 0)$ for any $\xi \in \Sigma$, and the flow $\sigma_{t}$ on $\Sigma^{f}$ is defined so that

$$
\sigma_{t}(\xi, s)=\left(\sigma^{k} \xi, u\right), \quad \text { if } \sum_{j=0}^{k-1} f\left(\sigma^{j} \xi\right) \leq t+s<\sum_{j=0}^{k} f\left(\sigma^{j} \xi\right),
$$

where $u=t+s-\sum_{j=0}^{k-1} f\left(\sigma^{j} \xi\right)$ and so on.

Theorem 1 and Theorem 2 follow from Proposition 3.1 and Proposition 3.2 below, respectively. 
Proposition 3.1. The map $h: \Sigma^{f} \rightarrow \Omega$ defined by $h(\xi, s)=S_{s}(x(\xi))$ gives the conjugacy between the flows $\sigma_{t}$ and $S_{t}$ restricted to $\Omega$ so that the corresponding closed orbits have the same period.

Proof. Theorem 0 in $\S 2$ implies that the map $h_{0}: \Sigma \rightarrow \Omega_{-}$defined by $h_{0}(\xi)=$ $x(\xi)$ is a homeomorphism with $h_{0}(\sigma \xi)=x(\sigma \xi)=T(x(\xi))=T\left(h_{0}(\xi)\right)$. Therefore $h_{0}$ gives a conjugacy between the Poincare maps $\sigma$ of $\sigma_{t}$ and $T$ of $S_{t}$ restricted to $\Omega$. On the other hand, the corresponding points $\xi$ and $h_{0}(\xi)$ have the same return time to $\Sigma$ and $\Omega_{-}$respectively from the definition of $f$. Hence $h$ gives a conjugacy between $\sigma_{t}$ and $S_{t}$ restricted to $\Omega$. Obviously the corresponding closed orbits have the same period.

Proposition 3.2. The ceiling function $f$ cannot be represented as

$$
f=g \circ \sigma-g+a K,
$$

where $g$ denotes a real valued function, $K$ an integer valued function, and $a$ positive constant.

Remark 3.1. Proposition 3.2 implies that the symbolic flow $\sigma_{t}$ is topologically weak mixing. On the other hand the estimate (2.4) shows that the celling function $f$ is Lipschitz continuous with respect to the metric $d_{\rho}$. Therefore we can obtain an analogue of the prime number theorem for the distribution of the prime closed orbits of the flow $S_{t}$ by use of the zeta function

$$
\begin{aligned}
\zeta(s) & =\exp \left(\sum_{n=1}^{\infty} \frac{1}{n} \sum_{\sigma^{n} \xi=\xi} \exp \left[-s \sum_{j=0}^{n-1} f\left(\sigma^{j} \xi\right)\right]\right) \\
& =\prod_{\gamma}\left(1-\exp \left[-s T_{\gamma}\right]\right)^{-1},
\end{aligned}
$$

where $\gamma$ denotes a prime closed orbit of $S_{t}$ and $T_{\gamma}$ is its period (see Parry and Pollicott [5]).

Proof of Proposition 3.2. Suppose that $f$ can be represented as in (3.3). By using the similarity transformation we may assume that $a=1$, i.e.,

$$
f=g \circ \sigma-g+K,
$$

where $g$ is a real valued function and $K$ is an integer valued function.

Since we already established the conjugacy in Proposition 3.1 we can identify the symbolic flow $\sigma_{t}$ and the flow $S_{t}$ restricted to $\Omega$ without confusion. The assumption (3.5) yields that every closed orbit of $S_{t}$ has an integer period. Now we restrict ourselves to three domains $O_{1}, O_{2}$, and $O_{3}$.

For each $n \geq 1$, let $\xi^{n}=\left(\xi_{j}^{n}\right)_{j=-\infty}^{\infty}$ be the sequence in $\sum$ so that $\xi_{0}^{n}=1$; $\xi_{j}^{n}=2$ for odd $j \leq 4 n-1 ; \xi_{j}^{n}=3$ for even $j \leq 4 n-2$; and $\xi_{m+4 n}^{n}=\xi_{m}^{n}$ for any $m \in Z$. Let $\xi^{0}$ be the sequence in with $\xi_{j}^{0}=3$ for even $j$ and $\xi_{j}^{n}=2$ for odd $j$. We denote by $x^{n, j}$ the unique element in $\Omega_{-}$which solves $\xi(x)=\sigma^{j} \xi^{n}$, and $q^{n, j}=\pi\left(x^{n, j}\right)$. Namely, $q^{n, j}$ denotes the position where the $j$ th collision 
occurs along the closed orbit $\gamma_{n}=\left(S_{t} x^{n, 0}\right)_{t}$ starting from $x^{n, 0}$, for $n \geq 0$. We note that the period $T_{n}$ of $\gamma_{n}$ has the minimal property which appeared in the proof of Theorem 0 (see the minimal value problem (2.11) in $\S 2$ ). The minimal property of $T_{n}$ and the uniqueness of the solution of the itinarary problem imply that $\gamma_{n}$ must by symmetric, i.e., $q^{n, 2 n+j}=q^{n, 2 n-j}$ for $j \geq 1$. We claim that

$$
T_{n+1} \geq T_{n}+2 T_{0}+1, \quad \text { for } n \geq 1 .
$$

Consider a fictitious motion of a particle such that the particle moves along the orbit $\gamma_{n+1}$ until it collides at $q^{n+1,2 n}$ and after that it returns to $q^{n+1,0}$ taking the same way as it has taken to reach $q^{n+1,2 n}$. It will be more convenient to introduce the following notation:

$$
q^{n+1,0} \rightarrow q^{n+1,1} \rightarrow \cdots \rightarrow q^{n+1,2 n} \rightarrow q^{n+1,2 n-1} \rightarrow \cdots \rightarrow q^{n+1,1} \rightarrow q^{n+1,0},
$$

where $p \rightarrow q$ denotes that the fictitious particle moves from $p$ to $q$.

Now we obtain a fictitious closed orbit $\gamma_{n}^{\prime}$ whose period $T_{n}^{\prime}$ is

$$
l\left(q^{n+1,0}, q^{n+1,1}, \ldots, q^{n+1,2 n}, q^{n+1,2 n-1}, \ldots, q^{n+1,1}\right) .
$$

Therefore, $T_{n}<T_{n}^{\prime}$ in virtue of the minimal value problem (2.11). Thus we have

$$
T_{n+1} \geq T_{n}^{\prime}+2 T_{0}>T_{n}+2 T_{0} .
$$

We used the fact that $\gamma_{n+1}$ is symmetric to see the first inequality in the above. But $T_{n}$ 's are all integers by our assumption. Hence we obtain (3.6).

On the other hand we can show

$$
T_{n+1} \leq T_{n}+2 T_{0}+C^{\prime} \rho^{2 n}
$$

where $C^{\prime}$ is a positive constant which is independent of $n$ and $\rho=(1+\eta)^{-1}$ as before. Clearly the inequality (3.7) contradicts the inequality (3.6). Therefore the celling function $f$ cannot be represented as in (3.5). This completes the proof of Proposition 3.2.

It remains to prove the inequality (3.7). We consider the following fictitious motion of a particle:

$$
\begin{aligned}
q^{n, 0} & \rightarrow q^{n, 1} \rightarrow \cdots \rightarrow q^{n, 2 n} \rightarrow q^{0,0} \rightarrow q^{0,1} \rightarrow q^{0,0} \rightarrow q^{n, 2 n} \\
& \rightarrow q^{n, 2 n-1} \rightarrow \cdots \rightarrow q^{n, 1} \rightarrow q^{n, 0} .
\end{aligned}
$$

Then we obtain the fictitious closed orbit $\gamma_{n+1}^{\prime}$ whose fictitious period $T_{n+1}^{\prime}$ is

$$
l\left(q^{n, 0}, q^{n, 1}, \ldots, q^{n, 2 n}, q^{0,0}, q^{0,1}, q^{0,1}, q^{n, 2 n}, q^{n, 2 n-1}, \ldots, q^{n, 1}\right) .
$$

On the other hand we have

$$
T_{n+1}^{\prime} \leq T_{n}+T_{0}+2\left|q^{n, 2 n}-q^{0,0}\right| \text {. }
$$

Here we used the fact that $\gamma$ is symmetric. From the definition of $\gamma_{n}$ and $\gamma_{0}, T^{j} x^{n, 2 n}$ and $T^{j} x^{0,1}$ belong to $\partial O_{\xi_{j+1}^{0}}$ for $|j| \leq 2 n-1$. Therefore the 
arclength $r\left(x^{0,1}, x^{n, 2 n}\right)$ between $q^{0,1}=\pi\left(x^{0,1}\right)$ and $q^{n, 2 n}=\pi\left(x^{n, 2 n}\right)$ is less than or equal to $C \rho^{2 n-1}$ by the a priori estimate $(2.5)$. Thus we obtain

$$
2\left|q^{n, 2 n}-q^{0,0}\right| \leq T_{0}+2 C \rho^{2 n-1}
$$

in virtue of the triangle inequality.

The inequalities (3.8) and (3.9) imply the inequality (3.7) in virtue of the minimal property of $T_{n+1}$.

Now the proof is complete.

Remark 3.2. In the proof of inequality (3.7) we used the fact that $\pi \gamma_{0}$ and $\pi \gamma_{n}$ cannot intersect. We restrict ourselves to note that it is an easy consequence of our hypotheses (H.1) and (H.2).

\section{REFERENCES}

1. V. Guillemin and R. Melrose, The poisson summation formula for manifold with boundary, Adv. in Math. 32 (1979), 204-232.

2. M. Ikawa, Singular perturbation of symbolic flows and poles of the zeta functions, preprint.

3. __ On the poles of the scattering matrix for several convex bodies, Proc. Japan Acad. Ser. A Math. Sci. 64 (1988), 91-93.

4. I. Kubo, Perturbed billiard system I, Nagoya Math. J. 61 (1976), 1-57.

5. W. Parry and M. Pollicott, An analogue of the prime number theorem for closed orbits of Axiom A flows, Ann. of Math. 118 (1983), 573-591.

6. Ya. G. Sinai, Dynamical systems with elastic reflections, Russian Math. Surveys 25 (1970), 137-189.

Department of Mathematics, Faculty of Science, Tokyo Institute of Technology, Oh-OKayama, Meguro-KU, Tokyo 152, JaPan 